disease (PD) could be improved if the bacterium is eradicated. It is hypothesized that $H$. pylori disrupts the primary site of levodopa absorption, and produces reactive oxygen species locally that could inactivate the drug.

Pierantozzi et al. studied the effects of $H$. pylori eradication in $H$. pylori-positive, motorfluctuating patients with PD on levodopa. Seventeen patients were given an $H$. pylori eradication treatment, of whom 15 were $H$. pylori-negative by study end; none of the 17 patients given a generic antioxidant changed H. pylori status. The eradication group showed a marked increase in levodopa absorption compared with the antioxidant group at both 2 weeks and 3 months, after both single and repeat administrations of levodopa. Improvements in clinical disability, 'on-time' duration, and remission of $H$. pylori-related gastroduodenitis, were also greater in the $H$. pylori eradication group, at both time points.

These results suggest that $H$. pylori interferes with clinical response to levodopa by impairing the drug's absorption-probably as a result of gastrointestinal alterations-but they do not support an effect related to production of reactive oxygen species. $H$. pylori eradication presents as an effective course of action in the large proportion of patients with PD who are infected with $H$. pylori.

Original article Pierantozzi M et al. (2006) Helicobacter pylori eradication and L-dopa absorption in patients with PD and motor fluctuations. Neurology 66: 1824-1829

\section{PLA2G6 mutations found in neurodegenerative disorders featuring high levels of brain iron}

A high level of iron in the brain is a feature associated with Parkinson's disease and Alzheimer's disease. High levels of brain iron also occur in patients with neurodegeneration with brain iron accumulation (NBIA) and in some patients with infantile neuroaxonal dystrophy (INAD). Researchers have recently investigated the molecular bases of these two neuroaxonal dystrophies, using genome-wide linkage studies in 12 families with INAD and a large consanguineous family with NBIA.

A locus for both INAD and NBIA was mapped to chromosome 22q12-q13. Mutations in the PLA2G6 gene (which encodes phospholipase $\mathrm{A}_{2}$, an enzyme that is critical in cell membrane homeostasis) were detected in both patients with INAD and those with NBIA, but were not present in matched controls. In total, 44 unique mutations were found, 32 of which were missense mutations; $85 \%$ of the missense mutations occurred at amino acid positions that are conserved in vertebrates. Brain MRI changes showing high levels of iron have not often been reported in INAD, but $40 \%$ of kindreds with mutation-positive INAD showed high brain iron in this study.

The authors conclude that defective phospholipid metabolism is implicated in neurodegenerative diseases featuring brain iron dyshomeostasis. This link between phospholipid defects and brain iron metabolism might lead to novel neuroprotective therapies.

Original article Morgan NV et al. (2006) PLA2G6, encoding a phospholipase $\mathrm{A}_{2}$, is mutated in neurodegenerative disorders with high brain iron. Nature Genet 38: 752-754

\section{Homer1a is a promising novel target for the treatment of inflammatory pain}

Synaptic glutamate receptors and the mobilization of intracellular calcium are important for nociceptive synaptic plasticity, and as such they constitute a potential target for the attenuation of inflammatory pain. The long-form Homer1 proteins are scaffold components that attach glutamate receptors to sources of calcium influx and release at synapses. Homer1a, however, is an endogenous, short, splice variant that lacks the ability to link glutamate receptors to synaptic proteins, and thus competitively dissociates synaptic glutamatergic signaling complexes.

In a recent study, Tappe et al. showed that peripheral inflammatory pain selectively upregulates Homer1a in the spinal cord neurons of rats. Using an RNA interference approach, the investigators generated mice in which this activity-induced upregulation of Homer1a was prevented. These mice showed an increase in spinal sensitization compared with control mice when subjected to a test for early postinflammatory hypersensitivity.

Calcium imaging of rat spinal slices injected with recombinant virions expressing Homer1a revealed a reduced calcium response to glutamate and a reduction in the density of synaptic spines on proximal dendrites when compared with control neurons. Nociceptive 